\title{
Alcoholic cardiomyopathy and liver disease in a community hospital in east Harlem in New York City
}

\begin{abstract}
Background: Alcohol abuse can result in alcoholic cardiomyopathy (ACM). As one of the most common causes of liver disease in patients admitted to our hospital is alcohol abuse, the aim of this study was to characterize patients with ACM and compare them to those with ischemic cardiomyopathy from a group of patients with heart failure (HF).
\end{abstract}

Methods: retrospective study of patients with HF defined as ejection fraction lower than $40 \%$, identified over a period of 5 years, from 2012 to 2017 . Data were analyzed by the use of T-test and Chi-square, as appropriate.

Results: 117 of 333(35\%) had ischemic cardiomyopathy (IC), and 216(64.8\%) had non ischemic cardiomyopathy (NICM); of these, 53(25\%) had ACM, which is 6.4\% of patients admitted with alcoholic liver disease (ALD). The majority of patients with ACM were of the black race, and was younger than those from the IC group.

Conclusion: ACM is not highly prevalent in the community of East Harlem, and cirrhosis is not commonly associated with ACM. Racial and genetic predisposition may play a role in the development of ACM.

Keywords: heart failure, pulmonary artery systolic pressure, left ventricular internal diameter in diastole, alanine transaminase, calcium channel blockers, left atrial diameter
Volume II Issue 4 - 2020

Tuoyo O Mene-Afejuku, ${ }^{1,2}$ Ahmed Shady, ${ }^{1,2}$ Adedoyin Akinlonu, ${ }^{1,2}$ Divya Nekkalapudi, ${ }^{1,2}$ Nora V Bergasa ${ }^{1,2,3}$

'Department of Internal Medicine, Woodhull Medical and Mental Health Center, USA

${ }^{2}$ Department of Internal Medicine, Metropolitan Hospital, USA ${ }^{3}$ Physician Affiliate Group of New York, USA

Correspondence: Nora V Bergasa, Department of Internal Medicine, Woodhull Medical and Mental Health Center, USA Tel 2I2-423-677I, Email Nora.Bergasa@icloud.com

Received: July II, 2020 | Published: August II, 2020
Abbreviations: BMI, body mass index; SBP, Systolic blood pressure; BUN, blood urea nitrogen; PASP, pulmonary artery systolic pressure; LVIDD, left ventricular internal diameter in diastole; LAD, left atrial diameter; AST, aspartate transaminase; ALT, alanine transaminase; ACE, angiotensin converting enzyme inhibitor; ARB, angiotensin receptor blocker; PSA, polysubstance abuse; CCB, calcium channel blockers

\section{Introduction}

Heart disease complicated by heart failure is a complication of cardiomyopathy, one of the causes being alcohol abuse disorder., ${ }^{1,2}$ Health + Hospitals/Metropolitan serves the community of East Harlem. ${ }^{3}$ Liver disease from alcohol is a prevalent diagnosis in the patients admitted to the hospital, a reflection of the data published in the community survey, in which alcohol related hospitalizations were 2.5 times greater than those in the rest of New York City. ${ }^{4}$ Similarly, admissions for heart disease in the community of East Harlem are
$40 \%$ greater than in the rest of New York City. ${ }^{4}$ The community needs assessment of 2016 identified heart disease and alcohol use as two of the most important health needs of the community. ${ }^{3}$ As there is an association between alcohol use disorder, and liver, and heart disease, ${ }^{1,2}$ the aim of this study was to characterize patients with alcoholic cardiomyopathy (ACM) and to compare them to those with ischemic cardiomyopathy (IC) from a group of patients with heart failure.

\section{Methods}

This was a retrospective study of 333 patients with HF defined as ejection fraction lower than $40 \%$, identified over a period of 5years, from 2012 to 2017. Patients with renal failure and those with cancer were excluded. Statistical analysis was done by Student's t test for continuous Over the study period, 817 patients with liver disease from alcohol variables, and Chi-square for categorical variables. The study was approved by the Institutional Review Board (Table 1).

Table I Baseline variables for heart failure secondary to ischemic cardiomyopathy vs heart failure secondary to alcoholic cardiomyopathy

\begin{tabular}{llll}
\hline Parameters & Ischemic cardiomyopathy & Alcoholic cardiomyopathy & p-value \\
\hline Age $($ years $)$ & $66.49 \pm|3.5|$ & $57.83 \pm 10.32$ & $0.000 I^{* *}$ \\
BMI $(\mathrm{Kg} / \mathrm{m})$ & $30.95 \pm 10.09$ & $28.09 \pm 6.70$ & $0.03 \mid$ \\
$\mathrm{SBP}(\mathrm{mmHg})$ & $|44.9| \pm 29.83$ & $136.23 \pm 29.72$ & 0.08 \\
Hemoglobin $(\mathrm{g} / \mathrm{dL})$ & $12.17 \pm 2.05$ & $12.50 \pm 1.89$ & 0.327 \\
Sodium(mmol/L) & $\mid 38.77 \pm 4.02$ & $138.23 \pm 4.87$ & 0.447 \\
BUN(mg/dL) & $24.85 \pm \mid 4.92$ & $24.68 \pm 15.87$ & 0.947 \\
\hline
\end{tabular}


Table Continued...

\begin{tabular}{llll}
\hline Parameters & Ischemic cardiomyopathy & Alcoholic cardiomyopathy & p-value \\
\hline Creatinine(mg/dL) & $1.61 \pm 1.38$ & $1.68 \pm 1.36$ & 0.763 \\
PASP (mmHg) & $37.13 \pm 14.18$ & $38.98 \pm 14.50$ & 0.453 \\
Length of stay(days) & $5.75 \pm 4.50$ & $4.38 \pm 2.85$ & $0.017^{* *}$ \\
LVIDD(cm) & $5.79 \pm 0.78$ & $6.08 \pm 1.12$ & 0.087 \\
LAD(cm) & $4.33 \pm 0.76$ & $4.68 \pm 0.61$ & $0.003^{* *}$ \\
Albumin (g/dL) & $3.43 \pm 2.05$ & $3.36 \pm 0.55$ & 0.814 \\
AST(units/L) & $44.89 \pm 39.22$ & $145.25 \pm 534.69$ & 0.182 \\
ALT(units/L) & $44.43 \pm 34.99$ & $93.87 \pm 216.69$ & 0.108 \\
Total bilirubin(mg/dL) & $1.40 \pm 7.10$ & $0.96 \pm 0.68$ & 0.658 \\
Direct bilirubin(mg/dL) & $0.28 \pm 0.33$ & $0.40 \pm 0.34$ & $0.044^{* *}$ \\
Liver disease & $14(12 \%)$ & $13(24.5 \%)$ & $0.038^{* *}$ \\
Gender (male) & $79(67.5 \%)$ & $44(83 \%)$ & $0.036^{* *}$ \\
Black race & $33(28.2 \%)$ & $24(45.3 \%)$ & $0.029^{* *}$ \\
ACE or ARB & $76(65 \%)$ & $44(83 \%)$ & $0.017^{* *}$ \\
PSA & $19(16.2 \%)$ & $21(39.6 \%)$ & $0.00 I^{* *}$ \\
Beta blocker(yes) & $82(70.1 \%)$ & $39(73.6 \%)$ & 0.641 \\
CCB(yes) & $24(20.5 \%)$ & $14(26.4 \%)$ & 0.392 \\
Spironolactone(yes) & $22(18.8 \%)$ & $10(18.9 \%)$ & 0.992 \\
Digoxin(yes) & $32(27.4 \%)$ & $16(30.2 \%)$ & 0.703 \\
Atrial fibrillation(yes) & $18(15.4 \%)$ & $9(17 \%)$ & 0.792 \\
\hline & & & \\
& & &
\end{tabular}

$* *=\mathrm{p} \leq 0.05$; BMI, body mass index; SBP, systolic blood pressure; BUN, blood urea nitrogen; PASP, pulmonary artery systolic pressure; LVIDD, left ventricular internal diameter in diastole; LAD, left atrial diameter;AST, aspartate transaminase; ALT, alanine transaminase; $A C E$, angiotensin converting enzyme inhibitor;ARB, angiotensin receptor blocker; PSA, polysubstance abuse; CCB, calcium channel blockers

\section{Results}

were admitted to the hospital. Of the 333 patients whose records were examined, 117(35\%) had ischemic cardiomyopathy (IC), and $216(64.8 \%)$ had non ischemic cardiomyopathy (NICM); of these, $53(25 \%)$ had ACM, 6.4\% of patients admitted with alcoholic liver disease (ALD). The characteristics of the patients with IC and those with ACM are shown in the table. The majority of the patients in both IC and ACM groups were of the male gender, $83 \%$ and $68 \%$, respectively. 24 patients $(45.3 \%)$ in the $\mathrm{ACM}$ were black, in contrast to $33(28 \%)$ of those in the IC group $(\mathrm{p}=0.029)$. The mean age of the group of patients with ACM was significantly less than that of patients with ICM, 58 \pm 10 years for the former, and $66 \pm 14$ for the latter $(p=0.0001)$, and the mean body mass index (BMI) was significantly higher in the NICM group, $31 \pm 10 \mathrm{~kg} / \mathrm{m}^{2}$, than that of the ACM group, $28 \pm 7 \mathrm{~kg} / \mathrm{m}^{2}(\mathrm{p}=0.031)$. Patients admitted for the management of heart failure from IC had a significantly longer length of stay, $6 \pm 5$ days, than those admitted with HF from ACM who stayed a mean of $4 \pm 3$ days $(p<0.017)$. Liver disease was significantly more prevalent in patients in the ACM group, $13(24.5 \%)$ patients, than in the group with IC, in which $14(12 \%)$ had this documented comorbidity $(\mathrm{p}=0.038)$; in this regard, serum concentration of direct bilirubin was significantly higher in the ACM.

\section{Discussion}

In this study of patients with heart failure, a quarter of the patients with NICM had ACM, and a quarter of those, the minority, had liver disease. The patients with ACM were younger than those with IC, the majority was of the male gender, and almost half were of the black race.

The proportion of patients with ACM in the population studied falls within the prevalence reported for patients with idiopathic dilated cardiomyopathy, $3.8 \%$ to $47 \%,{ }^{2,5-8}$ the lowest being from Baltimore, Maryland, in the United States, and the highest being from Europe. We had expected a higher prevalence of ACM that what was found based on the high proportion of patients of Hispanic ethnicity in East Harlem, 62\%, ${ }^{3}$ and the reported disparities in alcohol-related problems among white, black and Hispanic Americans in the United States. ${ }^{9}$ However, the majority of patients with ACM was of the black race. In addition, liver disease was documented in only a quarter of the patients with ACM, a difference from that reported from Spain, where cirrhosis was reported in $43 \%$ of patients with ACM.

The racial component and the relatively low prevalence of ACM in our study group may suggest that race and genetic susceptibility may determine the development of ACM. In the context of genetics, a 
variant of TTN, the gene that codes the sarcomere protein titin, TTNtv is being considered a prevalent genetic predisposition for ACM. ${ }^{10} \mathrm{It}$ was reported that variants in genes that have been well characterized as causes of dilated cardiomyopathy were more prevalent in patients with ACM than in a control group of subjects, although found with the same frequency in patients with ACM and dilated cardiomyopathy, and with an enhanced presence of titin truncating variants (TTNtv). In addition, an interaction between TTN genotype and excess alcohol consumption in a group of patients with dilated cardiomyopathy, not meeting criteria for ACM, was identified; furthermore, it was documented that patients with dilated cardiomyopathy with a TTNtv who excessively consumed alcohol had a significant decrease in the ejection fraction compared with those without gene variant and excess intake of alcohol. ${ }^{10}$ In the context of racial predisposition to develop ACM, robust epidemiological studies on ethnic and racial differences in the development of this cardiac complication have not been published, ${ }^{11}$ thus, we cannot provide pathophysiological insights on this particular result. However, the association of Titin variants and dilated cardiomyopathy in individual of African ancestry has been reported as weak. ${ }^{12}$

Liver disease was only documented in $24.5 \%$ of patients with ACM in our study group, with minimal hyperbilirubinemia noted in this group, possibly a reflection of some decrease in the hepatic excretory function. In this regard, the relationship between liver cirrhosis from alcohol in patients with ACM has been controversial; ${ }^{1}$ however, a prospective study documented that cirrhosis was present in $43 \%$ of patients with ACM. ${ }^{1}$ Although the interpretation of our results is very limited by the retrospective nature of our study, the fact a much lower prevalence of liver disease was found in our group may also reflect the genetic predisposition to develop cirrhosis from alcohol, and the difference between the populations studied, ours, from East Harlem, and the other one from Barcelona, Spain. ${ }^{1}$

\section{Conclusion}

Although ACM was not common as a cause of heart failure in hospitalized patients in our study, the importance to make the diagnosis, which is made on clinical grounds and by exclusion, is important because alcohol cessation is pivotal in the management of the patients with this complication of alcohol use disorder.

\section{Acknowledgments}

None.

\section{Conflicts of interest}

Author declares that there are no conflicts of interest.

\section{Funding}

None.

\section{References}

1. Estruch R, Fernandez-Sola J, Sacanella E, et al. Relationship between cardiomyopathy and liver disease in chronic alcoholism. Hepatology. 1995;22(2):532-538.

2. McKenna CJ, Codd MB, McCann HA, et al. Alcohol consumption and idiopathic dilated cardiomyopathy: a case control study. American Heart Journal. 1998;135(5 Pt 1):833-837.

3. $\mathrm{H}+\mathrm{H} /$ Metropolitan. Community health needs assessment. 2016.

4. Karpati A LX, Mostashari F, Thorpe L, et al. The Health of East Harlem. NYC Community Health Profiles. 2003;1(5):1-12.

5. Kasper EK, Agema WR, Hutchins GM, et al. The causes of dilated cardiomyopathy: a clinicopathologic review of 673 consecutive patients. J Am Coll Cardiol. 1994;23(3):586-590

6. Prazak P, Pfisterer M, Osswald S, et al. Differences of disease progression in congestive heart failure due to alcoholic as compared to idiopathic dilated cardiomyopathy. European Heart Journal. 1996;17(2):251-257.

7. Gavazzi A, De Maria R, Parolini M, Porcu M. Alcohol abuse and dilated cardiomyopathy in men. The American journal of cardiology. 2000 May 1;85(9):1114-8. PubMed PMID: 10781762.

8. Fauchier L, Babuty D, Poret P, et al. Comparison of long-term outcome of alcoholic and idiopathic dilated cardiomyopathy. European Heart Journal. 2000;21(4):306-314.

9. Mulia N, Ye Y, Greenfield TK, et al. Disparities in alcohol-related problems among white, black, and Hispanic Americans. Alcohol Clin Exp Res. 2009;33(4):654-662.

10. Ware JS, Amor-Salamanca A, Tayal U, et al. Genetic Etiology for Alcohol-Induced Cardiac Toxicity. Journal of the American College of Cardiology. 2018;71(20):2293-2302.

11. Piano MR, Phillips SA. Alcoholic cardiomyopathy: pathophysiologic insights. Cardiovascular toxicology. 2014;14(4):291-308.

12. Haggerty CM, Damrauer SM, Levin MG, et al. Genomics-First Evaluation of Heart Disease Associated With Titin-Truncating Variants. Circulation. 2019;140(1):42-54. 